\title{
AN IMMOBILIZATION TEST FOR AMOEBIASIS
}

\author{
BY \\ J. A. H. BROWN AND J. L. WHITBY \\ From the Royal Army Medical College, Millbank, London
}

(RECEIVED FOR PUBLICATION NOVEMBER 16, 1954)

The demonstration of antibodies in syphilitic sera that will immobilize Treponema pallidum (Nelson and Mayer, 1949) led Cole and Kent (1953) to investigate and demonstrate that immune rabbit sera were capable of immobilizing Entamoeba histolytica, and to suggest that sera from human cases of amoebiasis might show the same phenomenon. Their rabbit sera did not show immobilizing activity to a high titre but good immobilization of amoebae at single and twofold dilutions: further dilution of the sera led to a considerable fall in the immobilizing activity. In preliminary studies with sera from human cases of amoebiasis, these authors tested 13 sera, five of which showed some immobilizing activity in low dilution. The immobilizing effect appeared to be specific because 48 normal sera were without effect upon the amoebae.

A further laboratory test for the diagnosis of amoebiasis would clearly be desirable owing to the unsatisfactory nature of the complement-fixation test. In this paper the results of applying an immobilization technique in parallel with a complement-fixation test are described.

\section{Methods}

Complement-fixation Test. - Antigens were prepared from washed concentrated suspensions of threeday-old Entamoeba histolytica, from which alcoholic extracts were made by the method of Weiss and Arnold (1934). Control antigens were similarly prepared using fluid from the culture slopes.

Hyperimmune rabbit sera were used as controls. Cultures of amoebae were washed three times and concentrated to one-tenth volume, being suspended in physiological saline. After mixing with penicillin, $100 \mathrm{units} / \mathrm{ml}$., and streptomycin, $100 \mu \mathrm{g} . / \mathrm{ml}$., $0.5 \mathrm{ml}$. of the preparation was then inoculated intravenously into rabbits on alternate days, until a total of 10 injections had been given. The animals were bled after an interval of eight days.

Sera were inactivated at $56^{\circ} \mathrm{C}$. for 30 minutes. A unit volume of $0.1 \mathrm{ml}$. of diluted serum was used for each tube and an equal volume of 2.5 M.H.D. of pooled guinea-pig complement was added, followed by $0.1 \mathrm{ml}$. of antigen. The total volume of each tube was made up to $0.8 \mathrm{ml}$. with saline. Fixation was carried out at $37^{\circ} \mathrm{C}$. for one and half hours before adding $0.2 \mathrm{ml}$. sensitized sheep-cell system. This consisted of 5 M.H.D. haemolytic amboceptor in a $5 \%$ sheep-cell suspension. Tubes were placed in a $37^{\circ} \mathrm{C}$. water-bath for 30 minutes before reading.

Preliminary tests had shown that 2.5 M.H.D. of complement with one and a half hours' fixation at $37^{\circ} \mathrm{C}$. was superior to other periods of fixation and temperature. Particularly poor fixation was obtained at lower temperatures.

In the test, antigens were used at concentrations of 1 in 8 and 1 in 16 and antisera were usually tested at 1 in 4 and 1 in 8 .

Immobilization Test.-Amoebae were cultivated on Dobell and Laidlaw's (1926) modification of Boeck and Drbohlav (1925) medium. Subcultures from the sediment at the bottom of the slopes were made every three days for maintenance. Forty-eight-hour cultures were used for the immobilization test. A modified medium, in which $50 \%$ horse serum was substituted for 1 in 8 serum, in an endeavour to obtain growth free of rice starch, was tried, but as the yield of amoebae was very poor the method was abandoned.

All glassware used in the experiment was cleaned with acid.

The sediments from 48-hour-slope cultures of Entamoeba histolytica were pooled and allowed to stand for 30 minutes, after which time most of the supernatant fluid, which contained scanty amoebae, was removed. A concentrate containing between 200,000 and 400,000 amoebae $/ \mathrm{ml}$. was used for the test. Equal volumes of the serum to be tested and the amoebic suspension were mixed in a tube, and a drop of the mixture was transferred to a microscope slide and sealed with a coverslip and vaseline; all materials used in these manœuvres had been previously heated to $37^{\circ} \mathrm{C}$. The preparation, which was then maintained at $37^{\circ} \mathrm{C}$. in the incubator, was examined at intervals under the $2 / 3$ lens on a warmed stage, and the number of "rounded up" or immobile amoebae estimated. Twenty-five amoebae were counted and the result expressed as a percentage. "Non-rounded up," immobile amoebae were not regarded as immobilized, and the test was controlled by examining known positive and negative sera at the same time as the sera under test. The results were plotted as a curve, and sera showing more than $70 \%$ 
immobilization in the first hour were regarded as positive, those showing between $\mathbf{7 0} \%$ and $30 \%$ immobilization were considered doubtful, and those showing less than $30 \%$ immobilization negative.

\section{Results}

Immobilization is maximal at 30 minutes, and thereafter in most instances motility is gradually restored. In addition positive sera, which have not been inactivated by heat to destroy complement, may show lysis as well as immobilization.

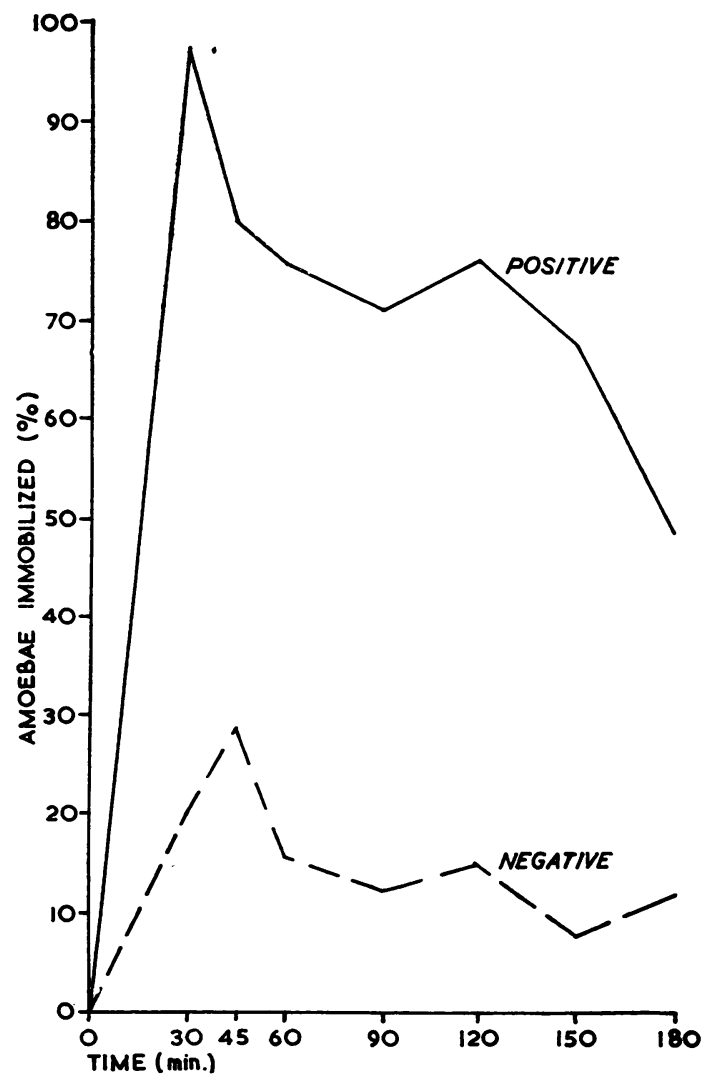

Fig. 1.-The curves shown are examples of the results obtained with typical positive and negative sera.

Of 25 sera tested, six were positive, three doubtful, and 16 negative by the immobilization test. The complement-fixation test was positive in three of the 25 sera tested, one of which was negative by the immobilization test. All sera tested were from active proved cases of amoebiasis save two, which were from old cases of amoebiasis with unproved but suspected recurrences. The latter sera were negative by both tests.

Fig. 1 illustrates typical findings with positive and negative sera.

\section{Discussion}

Immobilization of amoebae by human sera has $\stackrel{5}{?}$ been clearly demonstrated, but the very definite? results such as were obtained by Cole and Kent $\frac{\overline{0}}{\overline{5}}$. (1953) with hyperimmune rabbit sera have not been obtained by us even in severe cases of humano amoebiasis.

Amoebic dysentery has never been shown to be $-\overrightarrow{-}$ a disease stimulating a high level of antibody pro- $-\overrightarrow{-}$ duction in complement-fixation tests, and the inter- $\vec{\omega}$ pretation of these tests has never been very satis- $\frac{}{2}$ factory, as shown by Hussey and Brown (1950 to and Weiss and Arnold (1934). Therefore, it is hardly surprising that this apparent lack of anti-i genicity is reflected in immobilization tests. of

Heated inactivated sera showed immobilizingo activity of a temporary nature, and the immobil-ized amoebae gradually regained their activity after approximately one hour. This is in accord to ance with the observations of Cole and Kentos (1953). The action of complement is not required $\vec{\bullet}$ for this effect, and it has been shown that if com-cr plement is present lysis of the amoebae results. It is clear that immobilization tests are unlikely to be more satisfactory than complement-fixations tests owing to the considerable technical difficultiesō in maintaining the motility of the amoebae in the⿳⺈ controls and also because such tests appear to be@ little more sensitive than the present complement $-\overrightarrow{\vec{O}}$ fixation test.

It has not been possible in the present study to elucidate the nature of the immobilization reaction, but it seems that it is unrelated and probably fundamentally different from the reaction involving the use of complement. This matter appears to merit further investigation.

\section{Summary}

An immobilization test for amoebiasis has been described, and a comparison made with the com? plement-fixation test. The immobilization test was found to be little superior to the complement-o fixation test and involved considerably mores technical difficulty.

Our thanks are due to Colonel W. R. M. Drew, C.B.E., for much encouragement and advice and to? many pathologists who sent us specimens.

\section{REFERENCES} Boeck, W. C., and Drbohlav, J. (1925) Amer. J. Hyg., 5, 371.
Cole, B. A., and Kent, J. F. (1953). Proc. Soc. exp. Biol., N.Y., 83尺

Dobell, C., and Laidlaw, P. P. (1926). Parasitology, 18, 283. Hussey, K. L., and Brown, H. W. (1950). Amer. J. trop. Med. Hyg. 30147.

Nelson, R. A., and Mayer, M. M. (1949). J. exp. Med., 89, 369. Weiss, E., and Arnold, L. (1934). Amer. J. dig. Dis., 1, 231. 